\title{
Aliasing Error for Sampling Series Derivatives
}

\author{
R. M. Asharabi* \\ Institute of Mathematics, University of Lübeck, 23562 Lübeck, Germany \\ email: rashad1974@hotmail.com
}

\begin{abstract}
Elements of Bernstein spaces of band-limited functions with band width $\sigma, \sigma>0$ are perfectly sampled from their values at discrete set of points with different convergence criteria. The aliasing phenomenon occurs if the function is not band-limited or the sampling rate is lower than the bandwidth (Nyquist rate), i.e $\sigma \rightarrow \infty$. Since both conditions, band-limitedness and Nyquist rate are restrictive, it is desirable to find rigorous error estimates for the aliasing error. Here, several bounds for the aliasing error of sampling derivatives are established. We derive both uniform and $L_{p}$-norm bounds which are analogues of the results of Butzer et al. (2005), Fang (1996). Moreover, the so-called truncated aliasing error is investigated and a few numerical examples will be presented.
\end{abstract}

Key words and phrases: Sampling series, aliasing error, Fourier transform.

2010 AMS Mathematics Subject Classification —94A20, 41A05.

\section{Introduction}

For $1 \leq p<\infty, L_{p}(\mathbb{R})$ denotes the classical space of $p$-th Lebesgue integrable functions with the usual norm $\|\cdot\|_{p}$. Let $B_{\sigma, p}$ be the Bernstein space of all entire functions of exponential type $\sigma$ which belong to $L_{p}(\mathbb{R})$ when restricted to the real axis. According to Schwartz's theorem, cf. e.g. $[9,15]$

$$
B_{\sigma, p}:=\left\{f \in L_{p}(\mathbb{R}): \operatorname{supp} \widehat{f} \subset[-\sigma, \sigma]\right\},
$$

where in general $\widehat{f}(t)$ is the Fourier transform of $f(t)$ in the sense of generalized functions. For the special case $p=2, B_{\sigma, 2}$ is the well known Paley-Wiener space. The Whittaker-Kotelnikov-Shannon (WKS) sampling theorem states that every function $f \in B_{\sigma, 2}$ can be completely reconstructed from its sample values $f\left(\frac{k \pi}{\sigma}\right)$,

${ }^{*}$ On leave from Department of Mathematics, Faculty of Education Al-Mahweet, Sana'a University, Yemen. 
$k \in \mathbb{Z}$. In other words, if $f \in B_{\sigma, 2}$, then it can be expanded via the following sampling series

$$
f(t)=\sum_{k=-\infty}^{\infty} f\left(\frac{k \pi}{\sigma}\right) S_{k}(t), t \in \mathbb{R},
$$

where the function $S_{k}(t)$ is defined as

$$
S_{k}(t):=\operatorname{sinc}(\sigma t-k \pi)= \begin{cases}\frac{\sin (\sigma t-k \pi)}{(\sigma t-k \pi)}, & t \neq \frac{k \pi}{\sigma}, \\ 1, & t=\frac{k \pi}{\sigma} .\end{cases}
$$

The series (2) converges absolutely and uniformly on $\mathbb{R}$ and on compact subsets of $\mathbb{C}$, see e.g. $[9,15]$. Expansion $(2)$ is extended to the case $1 \leq p<\infty,[13,15]$. The phenomenon of aliasing occurs when the function is not band-limited, i.e $f \notin B_{\sigma, p}$, or when the sampling rate is lower than the band-width, i.e when $\sigma$ becomes bigger and bigger, or $\sigma \rightarrow \infty$. It is defined to be

$$
\mathcal{E}(f, t):=f(t)-\sum_{n=-\infty}^{\infty} f\left(\frac{n \pi}{\sigma}\right) S_{n}(t), t \in \mathbb{R} .
$$

Weiss [25] and Brown [12], to the best of our knowledge, were the first to derive bounds of aliasing error. Brown in [12] proved that, if $f \in L_{1}(\mathbb{R}) \cap C(\mathbb{R})$ and the Fourier transform $\widehat{f} \in L_{1}(\mathbb{R})$, then

$$
|\mathcal{E}(f, t)| \leq \sqrt{\frac{2}{\pi}} \int_{|w|>\sigma}|\widehat{f}(w)| d w, \quad t \in \mathbb{R}
$$

where $C(\mathbb{R})$ is the space of all uniformly continuous and bounded functions on $\mathbb{R}$. Estimate (5) bears appropriate characterization of error estimates of the aliasing error because the following properties

(PI) $\mathcal{E}(f, t)=0$ if $f \in B_{\sigma, 1}$.

(PII) It is the best possible in the sense that there is a function $f(t)$ which satisfies Browm's conditions such that

$$
|\mathcal{E}(f, t)|=\sqrt{\frac{2}{\pi}} \int_{|w|>\sigma}|\widehat{f}(w)| d w
$$

for some $t \in \mathbb{R}$, cf. [12].

(PIII) $\lim _{\sigma \rightarrow \infty} \mathcal{E}(f, t)=0$ uniformly on $\mathbb{R}$. 
There are several studies to extend Weiss-Brown result. Butzer and his collaborators work on this objective and they established estimate (5) for $f \in$ $L_{p}(\mathbb{R}) \cap C(\mathbb{R}), 1 \leq p \leq 2$ and $\widehat{f} \in L_{1}(\mathbb{R})$, as in [10]. In [7], Butzer et al. extended the results of $[10]$ to the case when $f \in L_{p}(\mathbb{R}) \cap C(\mathbb{R})$ and $\widehat{f} \in L_{1}(\mathbb{R}) \cap L_{q}(\mathbb{R})$ where $p, q>1$ and $1 / p+1 / q=1$. In another direction, Fang [13], also extended $(5)$ to $L_{p}(\mathbb{R})$ and other restrictive conditions. He first proved that if $f \in L_{p}(\mathbb{R})$, $1<p<\infty$ such that $\left\{f\left(\frac{n \pi}{\sigma}\right)\right\}_{n=-\infty}^{\infty} \in \ell_{p}$, then for every $g \in B_{\sigma, p}$ there is a constant $c_{p}$ which depends only on $p$ such that

$$
\|\mathcal{E}(f, t)\|_{p} \leq c_{p}\left(\frac{\pi}{\sigma} \sum_{n=-\infty}^{\infty}\left|f\left(\frac{n \pi}{\sigma}\right)-g\left(\frac{n \pi}{\sigma}\right)\right|^{p}\right)^{1 / p}+\|f-g\|_{p} .
$$

Here $\ell_{p}$ is the space of $p$-summable sequences with the standard norm. If in addition to the previous conditions, it is assumed that $f^{(r-1)} \in L_{p}(\mathbb{R}), 1<p<$ $\infty$, the set of all locally absolutely continuous functions on $\mathbb{R}$ and $f^{(r)} \in L_{p}(\mathbb{R})$, then cf. [13]

$$
\|\mathcal{E}(f, t)\|_{p} \leq c_{p, r} \sigma^{-r} \omega\left(f^{(r)}, 1 / \sigma\right)_{p}, \quad r \in \mathbb{N}, \sigma>1,
$$

where $c_{p, r}$, is the constant that depends only on $p, r$ and $\omega(f, t)_{p}$ is the modulus of continuity of $f$ in $L_{p}(\mathbb{R})$ which is defined as

$$
\omega(f, t)_{p}:=\sup _{|h| \leq t}\|f(\cdot+h)-f(\cdot)\|_{p} .
$$

Bardaro et al. introduced a subspace of $L_{p}(\mathbb{R})$ which allows one to estimate the aliasing error for functions which are not necessarily differentiable, see [2]. The studies of aliasing error are also extended to other types of sampling series, see e.g. $[3,11,16,17,18,19,22,26]$. Motivated by the results of Butser et al. and Fang mentioned above, we will study the following aliasing error

$$
\mathcal{E}_{r, \sigma}(f, t):=f^{(r)}(t)-\sum_{n=-\infty}^{\infty} f\left(\frac{n \pi}{\sigma}\right) S_{n}^{(r)}(t), t \in \mathbb{R}, r \in \mathbb{N}_{\circ},
$$

where $f^{(n)}(t):=\frac{d^{n}}{d t^{n}} f(t), n>0$ and $f^{(0)}(t):=f(t)$. Here $f$ belongs to some appropriate spaces and $\mathbb{N}_{\circ}$ denotes the set of non negative integers. To the best we know, except for the result of Stens [23] and of Butzer et al. [6], there are no studies on the aliasing error for the derivatives sampling theorem, i.e (9). Stens' result states that if $f \in L_{1}(\mathbb{R}) \cap C(\mathbb{R})$ and $w^{r} \widehat{f}(w) \in L_{1}(\mathbb{R})$, then

$$
\left|\mathcal{E}_{r, \sigma}(f, t)\right| \leq \sqrt{\frac{2}{\pi}} \int_{|w|>\sigma}\left|w^{r} \widehat{f}(w)\right| d w, \quad t \in \mathbb{R}, r \in \mathbb{N}_{\circ} .
$$

The main aim of this paper is to derive uniform and $L_{p}$ bounds for the aliasing error $\mathcal{E}_{r, \sigma}(f, t)$ when $f$ belongs to some spaces. This paper is divided up 
as follows. Section 2 contains the basic results which will be needed to prove the main results of this paper. We generalize some known results of mixed Hilbert transform and Whittaker operator in Section 3. Section 4 deals with uniform bounds and $L_{p}$-bounds for the aliasing error of sampling series derivatives. In the end of the Section 4, we derive bounds for so-called the truncated aliasing error. The final section contains two examples which illustrate the accuracy of the bounds.

\section{Preliminaries}

In this section we introduce some preliminary lemmas which we will use in the proofs of the main results of this paper. From now on, unless otherwise is stated, $r$ is a non negative integer and $\sigma>0$. For a given real parameter $t$, let $E_{t, r}(w)$ denote the periodic extension of the function $(i w)^{r} e^{i w t}$ on the interval $-\sigma<w \leq \sigma$ to the whole real line $\mathbb{R}$, i.e.

$$
E_{t, r}(w):=(i(w-2 \sigma k))^{r} e^{i(w-2 \sigma k) t}, \quad w \in[(2 k-1) \sigma,(2 k+1) \sigma), k \in \mathbb{Z} .
$$

Lemma 2.1. For any fixed $t \in \mathbb{R}$, we have

$$
E_{t, r}(w)=\sum_{n=-\infty}^{\infty} S_{n}^{(r)}(t) e^{i w n \pi / \sigma}, \quad w \in \mathbb{R}
$$

where $S_{n}(t)$ is defined in (3). The series (11) converges pointwise almost everywhere on $-\infty<w<\infty$. Also the partial sums of this Fourier series are bounded uniformly on $\mathbb{R}$ with respect $w$, i.e.

$$
\left|\sum_{n=-N}^{N} S_{n}^{(r)}(t) e^{i w n \pi / \sigma}\right| \leq C, \quad w \in \mathbb{R}, N \in \mathbb{N} .
$$

Proof. Fourier expansion (11) results directly from the computation of the Fourier coefficients

$$
a_{n}=\frac{1}{2 \sigma} \int_{-\sigma}^{\sigma}(i w)^{r} e^{i(t-n \pi / \sigma) w} d w=\frac{1}{2 \sigma} \frac{d^{r}}{d t^{r}} \int_{-\sigma}^{\sigma} e^{i(t-n \pi / \sigma) w} d w=S_{n}^{(r)}(t) .
$$

Convergence and boundedness results arise from [27, p. 90], see also [14, p. 32], and the fact $E_{t, r}(w)$ is periodic with bounded variation.

Consider the space

$$
\mathcal{F}^{p}:=\left\{f \in L_{p}(\mathbb{R}) \cap C(\mathbb{R}), \quad \widehat{f} \in L_{q}(\mathbb{R}) \cap L_{1}(\mathbb{R})\right\},
$$

where $1 \leq p<\infty, 1 / p+1 / q=1$ and $\widehat{f}$ denotes the Fourier transform of $f$, in the case $p \geq 2$ to be understood in the distributional seance. The condition $\widehat{f} \in L_{q}(\mathbb{R})$ in $(14)$ is always satisfied for $1 \leq p \leq 2$. The Fourier inversion formula of $f \in \mathcal{F}^{p}$ exists, [7]. 
Lemma 2.2. Let $f \in \mathcal{F}^{p}$ and $w^{r} \widehat{f}(w) \in L_{1}(\mathbb{R})$ and let

$$
F(t)=\frac{1}{\sqrt{2 \pi}} \int_{|w|>\sigma} \widehat{f}(w) e^{i w t} d w .
$$

The sampling series

$$
\sum_{k=-\infty}^{\infty} F\left(\frac{k \pi}{\sigma}\right) S_{k}^{(r)}(t)
$$

is convergent for all $t \in \mathbb{R}$ and can be written as

$$
\sum_{k=-\infty}^{\infty} F\left(\frac{k \pi}{\sigma}\right) S_{k}^{(r)}(t)=\frac{1}{\sqrt{2 \pi}} \sum_{0 \neq k \in \mathbb{Z}} \int_{(2 k-1) \sigma}^{(2 k+1) \sigma} \widehat{f}(w) E_{t, r}(w) d w, \quad t \in \mathbb{R} .
$$

Proof. From the definition of $F(t),(15)$, and the series in (11), we get

$$
\begin{aligned}
\lim _{N \rightarrow \infty} \sum_{k=-N}^{N} F\left(\frac{k \pi}{\sigma}\right) S_{k}^{(r)}(t) & =\frac{1}{\sqrt{2 \pi}} \lim _{N \rightarrow \infty} \sum_{k=-N}^{N}\left\{\int_{|w|>\sigma} \widehat{f}(w) e^{i w k \pi / \sigma} d w\right\} S_{k}^{(r)}(t) \\
& =\frac{1}{\sqrt{2 \pi}} \lim _{N \rightarrow \infty}\left\{\int_{|w|>\sigma} \widehat{f}(w) \sum_{k=-N}^{N} e^{i w k \pi / \sigma} S_{k}^{(r)}(t) d w\right\} \\
& =\frac{1}{\sqrt{2 \pi}} \int_{|w|>\sigma} \widehat{f}(w) \lim _{N \rightarrow \infty} \sum_{k=-N}^{N} e^{i w k \pi / \sigma} S_{k}^{(r)}(t) d w \\
& =\frac{1}{\sqrt{2 \pi}} \int_{|w|>\sigma} \widehat{f}(w) E_{t, r}(w) d w
\end{aligned}
$$

The interchange of sum and integral is justified from Lebesgue's dominated convergence theorem, noting that in view of (12)

$$
\left|\widehat{f}(w) \sum_{k=-N}^{N} e^{i w k \pi / \sigma} S_{k}^{(r)}(t)\right| \leq C|\widehat{f}(w)| \in L_{1}(\mathbb{R}), \quad N \in \mathbb{N} .
$$

This proves the first part of the lemma. The second part comes from (17) as following

$$
\begin{aligned}
\sum_{k=-\infty}^{\infty} F\left(\frac{k \pi}{\sigma}\right) S_{k}^{(r)}(t) & =\frac{1}{\sqrt{2 \pi}} \int_{|w|>\sigma} \widehat{f}(w) E_{t, r}(w) d w \\
& =\frac{1}{\sqrt{2 \pi}} \sum_{0 \neq k \in \mathbb{Z}} \int_{(2 k-1) \sigma}^{(2 k+1) \sigma} \widehat{f}(w) E_{t, r}(w) d w
\end{aligned}
$$


Lemma 2.3. Let $f \in \mathcal{F}^{p}$ and $w^{r} \widehat{f}(w) \in L_{1}(\mathbb{R})$. The sampling series

$$
\sum_{k=-\infty}^{\infty} f\left(\frac{k \pi}{\sigma}\right) S_{k}^{(r)}(t)
$$

is convergent for all $t \in \mathbb{R}$ and can be written as

$$
\sum_{k=-\infty}^{\infty} f\left(\frac{k \pi}{\sigma}\right) S_{k}^{(r)}(t)=\frac{1}{\sqrt{2 \pi}} \sum_{k \in \mathbb{Z}} \int_{(2 k-1) \sigma}^{(2 k+1) \sigma} \widehat{f}(w) E_{t, r}(w) d w, \quad t \in \mathbb{R} .
$$

Proof. First assume that $p \geq 2$. Since $f \in \mathcal{F}^{p}$, the Fourier inversion formula gives

$$
\begin{aligned}
f(t) & =\frac{1}{\sqrt{2 \pi}} \int_{-\infty}^{\infty} \widehat{f}(w) e^{i w t} d w \\
& =\frac{1}{\sqrt{2 \pi}} \int_{|w| \leq \sigma} \widehat{f}(w) e^{i w t} d w+\frac{1}{\sqrt{2 \pi}} \int_{|w|>\sigma} \widehat{f}(w) e^{i w t} d w \\
& =: G(t)+F(t)
\end{aligned}
$$

From (19) and (16), we get

$$
\begin{aligned}
\sum_{k=-\infty}^{\infty} f\left(\frac{k \pi}{\sigma}\right) S_{k}^{(r)}(t) & =\sum_{k=-\infty}^{\infty} G\left(\frac{k \pi}{\sigma}\right) S_{k}^{(r)}(t)+\sum_{k=-\infty}^{\infty} F\left(\frac{k \pi}{\sigma}\right) S_{k}^{(r)}(t) \\
& =\sum_{k=-\infty}^{\infty} G\left(\frac{k \pi}{\sigma}\right) S_{k}^{(r)}(t) \\
& +\frac{1}{\sqrt{2 \pi}} \sum_{0 \neq k \in \mathbb{Z}} \int_{(2 k-1) \sigma}^{(2 k+1) \sigma} \widehat{f}(w) E_{t, r}(w) d w
\end{aligned}
$$

The definition of $G(t)$ in (19) and the fact $\widehat{f} \in L_{q}(\mathbb{R})$ implies $G(t) \in B_{\sigma, p}$ and so the WKS can be applied to it. Thus

$$
G(t)=\sum_{k=-\infty}^{\infty} G\left(\frac{k \pi}{\sigma}\right) S_{k}(t)
$$

and the term by term differentiation of (21) given by

$$
G^{(r)}(t)=\sum_{k=-\infty}^{\infty} G\left(\frac{k \pi}{\sigma}\right) S_{k}^{(r)}(t)
$$

which converges uniformly on $\mathbb{R}$, see Remark 4.9 below. Because of $w^{r} \widehat{f}(w) \in$ $L_{1}(\mathbb{R})$, the function $G^{(r)}(t)$ can be written as

$$
G^{(r)}(t)=\frac{1}{\sqrt{2 \pi}} \int_{|w| \leq \sigma}(i w)^{r} \widehat{f}(w) e^{i w t} d w .
$$

Combining (20), (22), (23) implies (18) when $p \geq 2$. The equality (18) also true in the case $1 \leq p<2$ because of $\mathcal{F}^{p} \subset \mathcal{F}^{2}$ for all $p \in[1,2)$, see [7, p. 254]. 


\section{Generalized mixed Hilbert transform}

In this section, we introduce a generalized mixed Hilbert transform (MHT) which transforms the sequences $y_{k}$ from $\ell_{p}$ into $L_{p}(\mathbb{R})$. Let $K(t)$ be the unique integer satisfying $K(t)-1 / 2 \leq t<K(t)+1 / 2$, the MHT is defined to be

$$
\mathcal{H}_{j} y(t):=\sum_{k \in \mathbb{Z}, k \neq K(t)} \frac{y_{k}}{(t-k)^{j+1}}, \quad j \geq 0
$$

In the case $j=0$, Marsden [20] proved that $\mathcal{H}_{0}$ is bounded operator from $\ell_{p}$ into $L_{p}(\mathbb{R})$. Also Fang [17] proved the boundedness of $\mathcal{H}_{1}$. Here we study the case $j \geq 2$. From now we use the notations $\|\cdot\|_{L_{p}}$ and $\|\cdot\|_{\ell_{p}}$ for the norms of $L_{p}(\mathbb{R})$ and $\ell_{p}$ respectively. We state here the discrete version of Young's convolution inequality which will used in the proof of the next result. Let $1 \leq p, q<\infty$ such that $1 / p+1 / q=1 / r+1 \geq 0$. If $\left\{x_{k}\right\}_{k=1}^{\infty} \in \ell_{q}$ and $\left\{y_{k}\right\}_{k=1}^{\infty} \in \ell_{p}$ then the convolution $\left\{x_{k} * y_{k}\right\}_{k=1}^{\infty} \in \ell_{r}$ and

$$
\left\|x_{k} * y_{k}\right\|_{\ell_{r}} \leq\left\|x_{k}\right\|_{\ell_{q}}\left\|y_{k}\right\|_{\ell_{p}}
$$

cf. eg. [5, p. 266]. Taking the special case $p=r$, we get the following inequality

$$
\left\|x_{k} * y_{k}\right\|_{\ell_{p}} \leq\left\|x_{k}\right\|_{\ell_{1}}\left\|y_{k}\right\|_{\ell_{p}} .
$$

Lemma 3.4. The Hilbert transform $\mathcal{H}_{j}, j \geq 2$, is a bounded linear operator.

Proof. The linearity of $\mathcal{H}_{j}$ is clear. From the definition of $K(t)$, if $k \in \mathbb{Z}$ and $k \neq K(t)$, we have

$$
|K(t)-k| \leq|K(t)-x|+|t-k| \leq 1 / 2+|t-k|,
$$

which gives, for $t \neq k$,

$$
\left|\frac{K(t)-k}{t-k}\right| \leq \frac{1}{2|t-k|}+1 \leq 2 .
$$

Let

$$
\alpha(t):=\frac{(K(t)-t)(K(t)-k)}{t-k},
$$

then, we can write

$$
\frac{1}{t-k}=\frac{1}{K(t)-k}+\frac{\alpha(t)}{(K(t)-k)^{2}}, \quad t \neq k,
$$

and $|\alpha(t)| \leq 1$. Applying the binomial theorem, we get

$$
\frac{1}{(t-k)^{j+1}}=\sum_{m=0}^{j+1}\left(\begin{array}{c}
j+1 \\
m
\end{array}\right) \frac{\alpha^{j+1-m}(t)}{(K(t)-k)^{2 j+2-m}}, \quad j \geq 2 .
$$


Multiplying (28) by the sequence $y_{k}$ and taking the sum over all $k \in \mathbb{Z}, k \neq K(t)$, yields

$$
\begin{aligned}
\left|\mathcal{H}_{j} y(t)\right| & \leq \sum_{k \in \mathbb{Z}, k \neq K(t)} \sum_{m=0}^{j+1}\left(\begin{array}{c}
j+1 \\
m
\end{array}\right) \frac{\left|y_{k}\right|}{|K(t)-k|^{2 j+2-m}} \\
& \leq \sum_{m=0}^{j+1}\left(\begin{array}{c}
j+1 \\
m
\end{array}\right) \sum_{k \in \mathbb{Z}, k \neq K(t)} \frac{\left|y_{k}\right|}{|K(t)-k|^{2 j+2-m}},
\end{aligned}
$$

Taking the $L_{p}$-norm, $1<p<\infty$, to (29), we obtain

$$
\begin{aligned}
\left\|\mathcal{H}_{j} y(t)\right\|_{L_{p}} & \leq\left(\int_{-\infty}^{\infty}\left(\sum_{m=0}^{j+1}\left(\begin{array}{c}
j+1 \\
m
\end{array}\right) \sum_{k \in \mathbb{Z}, k \neq K(t)} \frac{\left|y_{k}\right|}{|K(t)-k|^{2 j+2-m}}\right)^{p} d t\right)^{1 / p} \\
& \leq \sum_{m=0}^{j+1}\left(\begin{array}{c}
j+1 \\
m
\end{array}\right)\left(\int_{-\infty}^{\infty}\left(\sum_{k \in \mathbb{Z}, k \neq K(t)} \frac{\left|y_{k}\right|}{|K(t)-k|^{2 j+2-m}}\right)^{p} d t\right)^{1 / p},
\end{aligned}
$$

where we have applied the Minkowsky's inequality $j+1$ times. Moreover

$$
\begin{gathered}
\int_{-\infty}^{\infty}\left(\sum_{k \in \mathbb{Z}, k \neq K(t)} \frac{\left|y_{k}\right|}{|K(t)-k|^{2 j+2-m}}\right)^{p} d t \\
=\sum_{n=-\infty}^{\infty} \int_{-1 / 2+n}^{1 / 2+n}\left(\sum_{k \in \mathbb{Z}, k \neq n} \frac{\left|y_{k}\right|}{|n-k|^{2 j+2-m}}\right)^{p} d t \\
=\sum_{n=-\infty}^{\infty}\left(\sum_{k \in \mathbb{Z}, k \neq n} \frac{\left|y_{k}\right|}{|n-k|^{2 j+2-m}}\right)^{p} .
\end{gathered}
$$

Substituting from (31) into (30), we get

$$
\left\|\mathcal{H}_{j} y(t)\right\|_{L_{p}} \leq \sum_{m=0}^{j+1}\left(\begin{array}{c}
j+1 \\
m
\end{array}\right)\left\|\sum_{k \in \mathbb{Z}, k \neq n} \frac{\left|y_{k}\right|}{|n-k|^{2 j+2-m}}\right\|_{\ell_{p}} .
$$

Applying Young's convolution inequality (26), yields

$$
\left\|\sum_{k \in \mathbb{Z}, k \neq n} \frac{\left|y_{k}\right|}{|n-k|^{2 j+2-m}}\right\|_{\ell_{p}} \leq \zeta_{m}\left\|y_{k}\right\|_{\ell_{p}}, \quad 0 \leq m \leq j+1 .
$$


where we have denoted by $\zeta_{m}$ to Riemann's zeta function, i.e

$$
\zeta_{m}:=\sum_{k=1}^{\infty} \frac{2}{k^{2 j+2-m}}
$$

Combining (33) and (32), implies

$$
\left\|\mathcal{H}_{j} y(t)\right\|_{L_{p}} \leq C_{j}\left\|y_{k}\right\|_{\ell_{p}}
$$

where

$$
C_{j}=\sum_{m=0}^{j+1} \zeta_{m}\left(\begin{array}{c}
j+1 \\
m
\end{array}\right)
$$

Hence $\mathcal{H}_{j}$ is bounded operator with $\left\|\mathcal{H}_{j}\right\|_{p} \leq C_{j}$.

We use the last result to prove that the Whittaker operator $\mathcal{L}_{\sigma}: \ell_{p} \rightarrow L_{p}(\mathbb{R})$ is bounded. It is defined as

$$
\mathcal{L}_{\sigma} y(t):=\sum_{k=-\infty}^{\infty} y_{k} S_{k}^{(r)}(t), \quad t \in \mathbb{R} .
$$

Theorem 3.5. Let $\left\{y_{k}\right\}_{k=-\infty}^{\infty} \in \ell_{p}, 1<p<\infty$. Then there exists a positive real number $\beta_{p, r}$ which depends on $p, r$ such that

$$
\left\|\mathcal{L}_{\sigma} y(t)\right\|_{L_{p}} \leq \beta_{p, r}\left(\frac{\pi}{\sigma}\right)^{1 / p}\left\|y_{k}\right\|_{\ell_{p}} .
$$

Proof. The case when $r=0$ is proved in [13]. Let $K(t)$ be as defined above. Then we get

$$
\left|\mathcal{L}_{\sigma} y(t)\right| \leq\left|\sum_{k \in \mathbb{Z}, k \neq K(t)} y_{k} S_{k}^{(r)}(t)\right|+\left|y_{K(t)} S_{K(t)}^{(r)}(t)\right|, \quad t \in \mathbb{R} .
$$

For convenience, we first consider the case $\sigma=\pi$. The derivatives of the sinc function is given by

$$
S_{k}^{(r)}(t)=\sigma^{r} \sum_{j=0}^{r}(-1)^{j}\left(\begin{array}{c}
r \\
j
\end{array}\right) j ! \frac{\sin (\sigma t-(k+(j-r) / 2) \pi)}{(\sigma t-k \pi)^{j+1}}, \quad t \neq \frac{k \pi}{\sigma},
$$

cf. [1, Lemma 2.1]. Using (38), (39) and the fact

$$
\left|S_{k}^{(r)}(t)\right| \leq \pi^{r},
$$

cf. e.g. [1], we obtain

$$
\left|\mathcal{L}_{\pi} y(t)\right| \leq \pi^{r}\left\{\sum_{j=0}^{r} j !\left(\begin{array}{c}
r \\
j
\end{array}\right)\left|\mathcal{H}_{j} y(t)\right|+\left|y_{K(t)}\right|\right\} .
$$


Noting that $y_{k} \in \ell_{p}$ and using the triangle inequality and (34), yield

$$
\begin{aligned}
\left\|\mathcal{L}_{\pi} y(t)\right\|_{L_{p}} & \leq \pi^{r}\left\{\sum_{j=0}^{r} j !\left(\begin{array}{c}
r \\
j
\end{array}\right)\left\|\mathcal{H}_{j} y(t)\right\|_{L_{p}}+\left\|y_{K(t)}\right\|_{L_{p}}\right\} \\
& \leq \pi^{r}\left\{\sum_{j=0}^{r} j !\left(\begin{array}{c}
r \\
j
\end{array}\right) C_{j}\left\|y_{k}\right\|_{\ell_{p}}+\left\|y_{K(t)}\right\|_{L_{p}}\right\} \\
& =\pi^{r}\left\{\sum_{j=0}^{r} j !\left(\begin{array}{c}
r \\
j
\end{array}\right) C_{j}+1\right\}\left\|y_{k}\right\|_{\ell_{p}},
\end{aligned}
$$

where we used that $\left\|y_{K(t)}\right\|_{L_{p}}=\left\|y_{k}\right\|_{\ell_{p}}$ which satisfied as following

$$
\left\|y_{K(t)}\right\|_{L_{p}}=\left(\sum_{k=-\infty}^{\infty} \int_{k-1 / 2}^{k+1 / 2}\left|y_{K(t)}\right|^{p} d t\right)^{1 / p}=\left(\sum_{k=-\infty}^{\infty}\left|y_{k}\right|^{p}\right)^{1 / p}
$$

By changing the scale, we obtain from (42) that

$$
\left\|\mathcal{L}_{\sigma} y(t)\right\|_{L_{p}} \leq \sigma^{r}\left(\frac{\pi}{\sigma}\right)^{1 / p}\left\{\sum_{j=0}^{r} j !\left(\begin{array}{c}
r \\
j
\end{array}\right) C_{j}\left(\frac{\pi}{\sigma}\right)^{j / p}+1\right\}\left\|y_{k}\right\|_{\ell_{p}}
$$

which is (37) where the constant $\beta_{p, r}:=\sigma^{r}\left\{\sum_{j=0}^{r} j !\left(\begin{array}{c}r \\ j\end{array}\right) C_{j}\left(\frac{\pi}{\sigma}\right)^{j / p}+1\right\}$ and $C_{j}$ is defined in (35).

\section{Main results}

This section contains the main results of this paper. In the following theorem we introduce the uniform bound of aliasing error

Theorem 4.6. Let $f \in \mathcal{F}^{p}$ and $w^{r} \hat{f}(w) \in L_{1}(\mathbb{R})$. Then we have for all $t \in \mathbb{R}$

$$
\left|\mathcal{E}_{r, \sigma}(f, t)\right| \leq \sqrt{\frac{2}{\pi}} \int_{|w|>\sigma}\left|w^{r} \hat{f}(w)\right| d w
$$

where the aliasing error, $\mathcal{E}_{r, \sigma}(f, t)$, is defined in (9). In particular,

$$
\lim _{\sigma \rightarrow \infty} \sum_{n=-\infty}^{\infty} f\left(\frac{n \pi}{\sigma}\right) S_{n}^{(r)}(t)=f^{(r)}(t)
$$

uniformly on $\mathbb{R}$. 
Proof. Since $f \in \mathcal{F}^{p}$, then the inversion Fourier transform is valid. Also since $w^{r} \widehat{f} \in L_{1}(\mathbb{R})$ for some $r \in \mathbb{N}$, then the $r$-th derivatives of $f$ exists as a continuous function, see e.g. [8, p. 197], and

$$
f^{(r)}(t)=\frac{1}{\sqrt{2 \pi}} \int_{-\infty}^{\infty}(i w)^{r} \widehat{f}(w) e^{i w t} d w, \quad t \in \mathbb{R} .
$$

Consequently

$$
f^{(r)}(t)=\frac{1}{\sqrt{2 \pi}} \sum_{k=-\infty}^{\infty} \int_{(2 k-1) \sigma}^{(2 k+1) \sigma}(i w)^{r} \widehat{f}(w) e^{i w t} d w, \quad t \in \mathbb{R} .
$$

Using Lemma 2.3, (18) and (48) and noting that $E_{t, r}(w)=(i w)^{r} e^{i w t}$ when $-\sigma \leq w<\sigma$, we obtain

$$
\begin{aligned}
\left|\mathcal{E}_{r, \sigma}(f, t)\right| & =\left|f^{(r)}(t)-\sum_{n=-\infty}^{\infty} f\left(\frac{n \pi}{\sigma}\right) S_{n}^{(r)}(t)\right| \\
& =\frac{1}{\sqrt{2 \pi}}\left|\sum_{k=-\infty}^{\infty} \int_{(2 k-1) \sigma}^{(2 k+1) \sigma}\left\{(i w)^{r} e^{i w t}-E_{t, r}(w)\right\} \widehat{f}(w) d w\right| \\
& =\frac{1}{\sqrt{2 \pi}}\left|\sum_{k \in \mathbb{Z}, k \neq 0} \int_{(2 k-1) \sigma}^{(2 k+1) \sigma}\left\{(i w)^{r} e^{i w t}-E_{t, r}(w)\right\} \widehat{f}(w) d w\right| \\
& \leq \frac{1}{\sqrt{2 \pi}} \sum_{k \in \mathbb{Z}, k \neq 0} \int_{(2 k-1) \sigma}^{(2 k+1) \sigma}\left|(i w)^{r} e^{i w t}-E_{t, r}(w)\right||\hat{f}(w)| d w .
\end{aligned}
$$

From the triangle inequality and the periodicity of $E_{t, r}(w)$, we get

$$
\left|(i w)^{r} e^{i w t}-E_{t, r}(w)\right| \leq\left|w^{r}\right|+\left|E_{t, r}(w)\right| \leq 2\left|w^{r}\right| .
$$

Substituting from (50) into (49) implies (45). The rest of the theorem arises from the fact that $\mathcal{E}_{r, \sigma}(f, t) \rightarrow 0$ as $\sigma \rightarrow \infty$ uniformly on $\mathbb{R}$.

Corollary 4.7. Let $f \in \mathcal{F}^{p}$ and $\hat{f}(w)=0$ for $|w|>\sigma$. Then

$$
f^{(r)}(t)=\sum_{n=-\infty}^{\infty} f\left(\frac{n \pi}{\sigma}\right) S_{n}^{(r)}(t), \quad t \in \mathbb{R}
$$

The series (51) converges absolutely and uniformly on $\mathbb{R}$.

Proof. Since $\hat{f}(w)=0$ for $|w|>\sigma$, then (45) implies (51) with uniformly convergence. 
Remark 4.8. The proof of uniformly convergence of (51) can be deduced with the help of the inequality

$$
\left(\sum_{k=-\infty}^{\infty}\left|S_{k}^{(r)}(t)\right|^{q}\right)^{1 / q}<(r+1) ! \sigma^{r} p, \quad t \in \mathbb{R},
$$

derived in $[1$, Lemma 2.4]. Indeed, let $p, q>1$ with $1 / p+1 / q=1$. Using (52) and the triangle and Hölder inequalities, we get

$$
\begin{aligned}
\left|f^{(r)}(t)-\sum_{k=-N}^{N} f\left(\frac{k \pi}{\sigma}\right) S_{k}^{(r)}(t)\right| & =\left|\sum_{|k|>N} f\left(\frac{k \pi}{\sigma}\right) S_{k}^{(r)}(t)\right| \\
& \leq\left(\sum_{|k|>N}\left|f\left(\frac{k \pi}{\sigma}\right)\right|^{p}\right)^{1 / p}\left(\sum_{|k|>N}\left|S_{k}^{(r)}(t)\right|^{q}\right)^{1 / q}, \\
& \leq(r+1) ! \sigma^{r} p\left(\sum_{|k|>N}\left|f\left(\frac{k \pi}{\sigma}\right)\right|^{p}\right)^{1 / p} .
\end{aligned}
$$

Since $f \in B_{\sigma, p}$, then there exists a constant $\alpha_{p}$ such that, [24, p. 233],

$$
\left(\frac{\pi}{\sigma} \sum_{k=-\infty}^{\infty}\left|f\left(\frac{k \pi}{\sigma}\right)\right|^{p}\right)^{1 / p} \leq \alpha_{p}\|f\|_{L_{p}}<\infty,
$$

which implies that $\left\{f\left(\frac{k \pi}{\sigma}\right)\right\}_{n=-\infty}^{\infty} \in \ell_{p}$. Since the bound in (53) is independent of $t$ and tends to zero as $N \rightarrow \infty$, the convergence is established.

\section{Remark 4.9. .}

1. The assumption in Corollary 4.7 equivalent that $f \in B_{\sigma, p}$.

2. If we let $r=0$ and $p=1$ in (45), (46) and (51), then we get the results of Brown, [12].

3. If we let $r=0$ in (45), (46) and (51), then we get the results of Butzer and Stens, [10].

In the next result we proved that the sampling series derivatives converges in the sense $L_{p}$-norm.

Theorem 4.10. Let $f \in B_{\sigma, p}, 1<p<\infty$. Then

$$
\begin{aligned}
& \text { (a) } \lim _{n \rightarrow \infty}\left\|f^{(r)}(t)-\sum_{k=-n}^{n} f\left(\frac{k \pi}{\sigma}\right) S_{k}^{(r)}(t)\right\|_{L_{p}}=0, \\
& \text { (b) }\left\|f^{(r)}\right\|_{L_{p}} \leq \beta_{p, r}\left(\frac{\pi}{\sigma} \sum_{k=-\infty}^{\infty}\left|f\left(\frac{k \pi}{\sigma}\right)\right|^{p}\right)^{1 / p},
\end{aligned}
$$

where $\beta_{p, r}$ is a positive constant which depends only on $p, r$. 
Proof. Since $f \in B_{\sigma, p}, 1 \leq p<\infty$, then (51) is satisfies, [15, Theorem 6.14], and $\left\{f\left(\frac{k \pi}{\sigma}\right)\right\}_{n=-\infty}^{\infty} \in \ell_{p},[4$, Theorem 6.7.1]. Using (51) and (37), we get

$$
\begin{aligned}
\left\|f^{(r)}(t)-\sum_{k=-n}^{n} f\left(\frac{k \pi}{\sigma}\right) S_{k}^{(r)}(t)\right\|_{L_{p}} & =\left\|\sum_{|k|>n} f\left(\frac{k \pi}{\sigma}\right) S_{k}^{(r)}(t)\right\|_{L_{p}} \\
& \leq \beta_{p, r}\left(\frac{\pi}{\sigma} \sum_{|k|>n}\left|f\left(\frac{k \pi}{\sigma}\right)\right|^{p}\right)^{1 / p} .
\end{aligned}
$$

The right hand-sid of (57) tends to zero when $n \rightarrow \infty$. Thus the proof of (55) completed. Applying (51) and (37), implies immediately (56).

Remark 4.11. If we let $r=0$ in (55) and (56), then we get the results of Fang, [13].

Denote by $L_{p}^{r}(\mathbb{R}), 1 \leq p<\infty, r \in \mathbb{N}$, the subspace of functions $f \in L_{p}(\mathbb{R})$ which the $(r-1)$-th derivative of $f$ exists and is locally absolutely continuous on $\mathbb{R}$, and $f^{(r)} \in L_{p}(\mathbb{R})$. It is clear that $B_{\sigma, p} \subset L_{p}^{r}(\mathbb{R})$. In the following result we introduce a bound of aliasing error when $f \in L_{p}^{r}(\mathbb{R}), 1<p<\infty$.

Theorem 4.12. Let $f \in L_{p}^{r}(\mathbb{R}), 1<p<\infty$ and $r \in \mathbb{N}$. Then for every $g \in B_{\sigma, p}$, we have

$$
\left\|\mathcal{E}_{r, \sigma}(f, t)\right\|_{p} \leq \beta_{p, r}\left(\frac{\pi}{\sigma}\right)^{1 / p}\left\|f\left(\frac{k \pi}{\sigma}\right)-g\left(\frac{k \pi}{\sigma}\right)\right\|_{\ell_{p}}+\left\|f^{(r)}-g^{(r)}\right\|_{L_{p}} .(58)
$$

where $\mathcal{E}_{r, \sigma}(f, t)$ is defined in $(9)$.

Proof. Let $g \in B_{\sigma, p}, 1<p<\infty$. Then $g$ has the following sampling series derivatives

$$
g^{(r)}(t)=\sum_{k=-\infty}^{\infty} g\left(\frac{k \pi}{\sigma}\right) S_{k}^{(r)}(t), \quad r \in \mathbb{N},
$$

Using (59) and the triangle inequality, we obtain

$$
\left\|\mathcal{E}_{r, \sigma}(f, t)\right\|_{L_{p}} \leq\left\|\sum_{k=-\infty}^{\infty}\left\{f\left(\frac{k \pi}{\sigma}\right)-g\left(\frac{k \pi}{\sigma}\right)\right\} S_{k}^{(r)}(t)\right\|_{L_{p}}+\left\|f^{(r)}-g^{(r)}\right\|_{L_{p}} .(60
$$

Since $f \in L_{p}^{r}(\mathbb{R})$, then the sequence $\{f(k \pi / \sigma)\}_{k \in \mathbb{Z}} \in \ell_{p}$, [13, Lemma 3.4]. Also $\{g(k \pi / \sigma)\}_{k \in \mathbb{Z}} \in \ell_{p}$ because of $g \in B_{\sigma, p}$. Therefore we can use (37) to get

$$
\left\|\sum_{k=-\infty}^{\infty}\left\{f\left(\frac{k \pi}{\sigma}\right)-g\left(\frac{k \pi}{\sigma}\right)\right\} S_{k}^{(r)}(t)\right\|_{L_{p}} \leq \beta_{p, r}\left(\frac{\pi}{\sigma}\right)^{1 / p}\left\|f\left(\frac{k \pi}{\sigma}\right)-g\left(\frac{k \pi}{\sigma}\right)\right\|_{\ell_{p}}
$$

Substituting from (61) into (60) yields (58). 
Let $\Delta_{h}^{k} f(x):=\sum_{j=0}^{k} C_{j}^{k} f(x+j h)$ be the $k$-th difference as a measure of the smoothness of the functions where $C_{j}^{k}$ is binomial coefficient. We use the modulus of the continuity with respect

$$
\omega_{k}(f, t)_{p}:=\sup _{|h| \leq t}\left\|\Delta_{h}^{k} f(\cdot)\right\|_{L_{p}} .
$$

In the next result we get a bound of aliasing error under the assumptions of Theorem 4.12 and the restricted $\sigma>1$.

Theorem 4.13. Let $f \in L_{p}^{r}(\mathbb{R}), 1<p<\infty, r \in \mathbb{N}$ and $\sigma>1$. Then there is a constant $M_{p, r}$ which depends only on $p$ and $r$ such that

$$
\left\|\mathcal{E}_{r, \sigma}(f, t)\right\|_{L_{p}} \leq M_{p, r} \sigma^{-r} \omega\left(f^{(r)}, 1 / \sigma\right)_{p} .
$$

Proof. Let $1<p<\infty$, and $g \in B_{\sigma, p}$. Then $g \in L_{p}(\mathbb{R})$ and it has an infinitely differentiable which belong to $L_{p}(\mathbb{R})$. Since $f, g \in L_{p}^{(r)}(\mathbb{R})$, then we have, $[13$, Lemma 3.4],

$$
\left(\frac{\pi}{\sigma}\right)^{1 / p}\left\|f\left(\frac{k \pi}{\sigma}\right)-g\left(\frac{k \pi}{\sigma}\right)\right\|_{\ell_{p}} \leq\|f-g\|_{L_{p}}+\frac{\pi}{\sigma}\left\|f^{\prime}-g^{\prime}\right\|_{L_{p}}<\infty .
$$

Combining between (58) and (64), we get for every $g \in B_{\sigma, p}$

$$
\left\|\mathcal{E}_{r, \sigma}(f, t)\right\|_{L_{p}} \leq \beta_{p, r}\left(\|f-g\|_{L_{p}}+\frac{\pi}{\sigma}\left\|f^{\prime}-g^{\prime}\right\|_{L_{p}}\right)+\left\|f^{(r)}-g^{(r)}\right\|_{L_{p}} .
$$

Because of $f \in L_{p}^{r}(\mathbb{R}), 1<p<\infty$, we have [24, p. 257]

$$
\left\|f^{(j)}-g_{\sigma}^{(j)}\right\|_{L_{p}} \leq A_{k} \omega_{k}\left(f^{(j)}, 1 / \sigma\right)_{p}, \quad j=0,1, \ldots r,
$$

for every integer $k$, [24, p. 257]. Substituting from (66) into (65) yields

$$
\begin{aligned}
\left\|\mathcal{E}_{r, \sigma}(f, t)\right\|_{L_{p}} \leq & \beta_{p, r} A_{r+1}\left\{\omega_{r+1}(f, 1 / \sigma)_{p}+\pi \sigma^{-1} \omega_{r+1}\left(f^{\prime}, 1 / \sigma\right)_{p}\right\} \\
& +A_{r+1} \omega_{r+1}\left(f^{(r)}, 1 / \sigma\right)_{p} .
\end{aligned}
$$

Letting $\sigma>1$ and using the following properties of modulus of smoothness, [24, p. 102-104],

$$
\begin{gathered}
\omega_{r+1}(f, 1 / \sigma)_{p} \leq \sigma^{-r} \omega\left(f^{(r)}, 1 / \sigma\right)_{p}, \\
\omega_{r+1}\left(f^{\prime}, 1 / \sigma\right)_{p} \leq 2 \sigma^{-r+1} \omega\left(f^{(r)}, 1 / \sigma\right)_{p}, \\
\omega_{r+1}\left(f^{(r)}, 1 / \sigma\right)_{p} \leq 2^{r} \omega\left(f^{(r)}, 1 / \sigma\right)_{p},
\end{gathered}
$$

where $r \in \mathbb{Z}^{+}$, the inequality (67) becomes (63) and the proof is completed. 
It is clear from (58) that the bound of aliasing error tends to zero when $f \in B_{\sigma, p}$. The important question which discuses in the next result, does the aliasing error converge in the sense of $L_{p}$-norm as $\sigma \rightarrow \infty$ ?

Corollary 4.14. Let $f \in L_{p}^{r}(\mathbb{R}), 1<p<\infty, r \in \mathbb{N}_{\circ}$. Then we have

$$
\lim _{\sigma \rightarrow \infty}\left\|\mathcal{E}_{r, \sigma}(f, t)\right\|_{L_{p}}=0
$$

Proof. When $r=0$ the equality (71) becomes directly from [13, Theorem 5]. If $r \geq 1$, it becomes from (63) by taking $\sigma \rightarrow \infty$ and noting that $\lim _{\sigma \rightarrow \infty} \omega(f, 1 / \sigma)_{p}=$ 0 .

The bounds of aliasing error in (45), (58) and (63) requires us to know the exact values of $f$ at the infinity points $\left\{\frac{k \pi}{\sigma}\right\}_{k=-\infty}^{\infty}$ and the sum of an infinite series. In practical situations, only finitely many samples are available so in the next results we introduce bounds for the so-called truncated aliasing error. For any $N \in \mathbb{Z}^{+}$we consider the finite sum

$$
T_{N}[f](t)=\sum_{k=-N}^{N} f\left(\frac{k \pi}{\sigma}\right) S_{k}(t)
$$

The truncated aliasing error is defined as $\left|f^{(r)}(t)-T_{N}^{(r)}[f](t)\right|$. The following results are devoted to an uniform and $L_{p}$ bounds for the truncated aliasing error.

Theorem 4.15. Let $f \in \mathcal{F}^{p}, w^{r} \hat{f}(w) \in L_{1}(\mathbb{R})$ and $\{f(k \pi / \sigma)\}_{k \in \mathbb{Z}} \in \ell_{p}$. Then for all $N \in \mathbb{Z}^{+}$, we have

$$
\begin{aligned}
\left|f^{(r)}(t)-T_{N}^{(r)}[f](t)\right| \leq & \sqrt{\frac{2}{\pi}} \int_{|w|>\sigma}\left|w^{r} \hat{f}(w)\right| d w \\
& +\sigma^{r}(r+1) !\left(\sum_{|k|>N}\left|f\left(\frac{k \pi}{\sigma}\right)\right|^{p}\right)^{1 / p} .
\end{aligned}
$$

Proof. Using the triangle inequality, we obtain

$$
\left|f^{(r)}(t)-T_{N}^{(r)}[f](t)\right| \leq\left|\mathcal{E}_{r, \sigma}(f, t)\right|+\sum_{|k|>N}\left|f\left(\frac{k \pi}{\sigma}\right) S_{k}^{(r)}(t)\right| .
$$

Let $p, q>1$ such that $1 / p+1 / q=1$. Applying Hölder's inequality and using 
(52) yields

$$
\begin{aligned}
\sum_{k \notin \mathbb{Z}_{N}(t)}\left|f\left(\frac{k \pi}{\sigma}\right) S_{k}^{(r)}(t)\right| & \leq\left(\sum_{|k|>N}\left|f\left(\frac{k \pi}{\sigma}\right)\right|^{p}\right)^{1 / p}\left(\sum_{|k|>N}\left|S_{k}^{(r)}(t)\right|^{q}\right)^{1 / q} \\
& \leq \sigma^{r}(r+1) !\left(\sum_{|k|>N}\left|f\left(\frac{k \pi}{\sigma}\right)\right|^{p}\right)^{1 / p}<\infty,
\end{aligned}
$$

in view of $\{f(k \pi / \sigma)\}_{k \in \mathbb{Z}} \in \ell_{p}$. The bound (73) arises by combining (45), (74) and (75).

Theorem 4.16. Let $f \in L_{p}^{r}(\mathbb{R}), 1<p<\infty$. Then for all $N \in \mathbb{N}$ and $\sigma>1$ there exist constants $M_{p, r}$ and $\beta_{p, r}$ such that

$$
\begin{aligned}
\left\|f^{(r)}(t)-T_{N}^{(r)}[f](t)\right\|_{L_{p}} \leq & M_{p, r} \sigma^{-r} \omega\left(f^{(r)}, 1 / \sigma\right)_{p} \\
& +\beta_{p, r}\left(\frac{\pi}{\sigma} \sum_{|k|>N}\left|f\left(\frac{k \pi}{\sigma}\right)\right|^{p}\right)^{1 / p},
\end{aligned}
$$

where $r \in \mathbb{N}$ and $\omega$ is defined in (8).

Proof. Since $f \in L_{p}^{(r)}(\mathbb{R})$, then, as we see in (64), the sequence $\left\{f\left(\frac{k \pi}{\sigma}\right)\right\}_{k=-\infty}^{\infty} \in$ $\ell_{p}$. Using the triangle inequality and (37), we get

$$
\begin{aligned}
\left\|f^{(r)}(t)-T_{N}^{(r)}[f](t)\right\|_{L_{p}} & \leq\left\|\mathcal{E}_{r, \sigma}(f, t)\right\|_{L_{p}}+\left\|\sum_{|k|>N} f\left(\frac{k \pi}{\sigma}\right) S_{k}^{(r)}(t)\right\|_{L_{p}} \\
& \leq\left\|\mathcal{E}_{r, \sigma}(f, t)\right\|_{L_{p}}+\beta_{p, r}\left(\frac{\pi}{\sigma} \sum_{|k|>N}\left|f\left(\frac{k \pi}{\sigma}\right)\right|^{p}\right)^{1 / p} .
\end{aligned}
$$

Combining (77) and (63) implies (76)

\section{$5 \quad$ Examples}

This section contains two simple examples whose show the accuracy of the truncated series and the accuracy of the uniform bound in (73). In the following examples we select the function $f$ to be a strictly decreasing on $[N, \infty)$ therefore we compute the infinite sequence in (73) as following

$$
\sum_{|k|>N}\left|f\left(\frac{k \pi}{\sigma}\right)\right|^{p}<\frac{2 \sigma}{\pi} \int_{N \pi / \sigma}^{\infty}|f(t)|^{p} d t .
$$

Through this section we denote to the point $t_{j}:=\left(j-\frac{1}{2}\right) \frac{\pi}{\sigma}, j \in \mathbb{N}$. 
Example 5.17. Consider the function

$$
f(t)=\sin (t) e^{-|t|},
$$

which is in $L_{2}(\mathbb{R})$ and its Fourier transform belongs to $L_{1}(\mathbb{R})$

$$
\widehat{f}(w)=-\sqrt{\frac{2}{\pi}} \frac{2 i w}{4+w^{4}} .
$$

This function and its Fourier transform satisfy the conditions of Theorem 4.10. The accuracy of the truncated series and the bound will be shown in the following table where $r=0$ and $N:=\lfloor 2 \sigma\rfloor$.

\begin{tabular}{c|c|c|c}
\hline$\sigma$ & $t$ & absolute error & uniform bound \\
\hline \multirow{5}{*}{$10 \pi$} & $t_{1}$ & $6.8280 \times 10^{-4}$ & \\
& $t_{2}$ & $2.1801 \times 10^{-4}$ & \\
& $t_{3}$ & $1.0065 \times 10^{-4}$ & $3.5977 \times 10^{-3}$ \\
& $t_{4}$ & $5.6498 \times 10^{-5}$ & \\
\hline
\end{tabular}

Example 5.18. The Gauss function

$$
f(t)=e^{-t^{2} / 2}, \quad t \in \mathbb{R},
$$

has the Fourier transform

$$
\widehat{f}(w)=e^{-w^{2} / 2}, \quad w \in \mathbb{R} .
$$

We apply Theorem 4.10 to exhibit the accuracy of bound when $p=2, r=2$ and $N:=\lfloor 2 \sigma\rfloor$

\begin{tabular}{c|c|c|c}
\hline$\sigma$ & $t$ & absolute error & uniform bound \\
\hline \multirow{5}{*}{$2 \pi$} & $t_{1}$ & $9.4866 \times 10^{-10}$ & \\
& $t_{2}$ & $2.8120 \times 10^{-9}$ & \\
& $t_{3}$ & $4.5761 \times 10^{-9}$ & $7.1711 \times 10^{-7}$ \\
& $t_{4}$ & $6.1843 \times 10^{-9}$ & \\
\hline
\end{tabular}

\section{ACKNOWLEDGEMENT}

The author would like to thank Professor M.H. Annaby for his comments and critical reading of the manuscript and Professor J. Prestin for hospitality during his postdoctoral-periodic in the Institute of Mathematics, University of Lübeck, Germany, 2012-2013. He also gratefully acknowledges the support by the Alexander von Humboldt foundation under the grant 3.4-JEM/142916. 


\section{References}

[1] M. H. Annaby and R. M. Asharabi, Truncation, amplitude and jitter errors on $\mathbb{R}$ for sampling series derivatives, J. Approx. Theory 163 (2011), 336362 .

[2] C. Bardaro, P. L. Butzer, R. L. Stens and G. Vinti, Approximation error of the Whitteker cardinal series in terms of an averaged modulus of smoothness covering discontinuous signals, J. Math. Anal. Appl. 316 (2006), 269-306.

[3] M. G. Beaty and J. R. Higgins, Aliasing and Poisson summation in the sampling theory of Paley-Wiener spaces, J. Fourier Anal. Appl. 1 (1994), $67-85$.

[4] R. P. Boas, Entire Functions, Academic Press, New Yourk, 1954.

[5] P. S. Bullen, A Dictionary of Inequality, Pitman Monographs and Surveys in Pure and Applied Mathematics 97, Longman, Harlow, 1998.

[6] P. Butzer, G. Schmeisser, and R. Stens, Shannon's sampling theorem for bandlimited signals and their Hilbert transform, Boas-type formulae for higher order derivatives-The aliasing error involved by their extensions from bandlimited to non-bandlimited signals, J. Entropy, 14 (2012), 2192-2226.

[7] P. L. Butzer, J. R. Higgins and R. L. Stens, Classical and approximate sampling theorems; studies in the $L^{p}(\mathbb{R})$ and the uniform norm J. Approx. Theory, 137 (2005), 250-263.

[8] P. L. Butzer and R. J. Nessel, Fourier Analysis and Approximation, Academic Press, New Yourk, 1971.

[9] P. L. Butzer, G. Schmeisser and R. L. Stens, An introduction to sampling analysis, In: Non Uniform Sampling: Theory and Practices F. Marvasti (ed), Kluwer, New York (2001), 17-121.

[10] P. L. Butzer and R. L. Stens, The Euler-MacLaurin summation formula, the sampling theorem, and approximate integration over the real axis, Linear Algebra Appl. 52/53 (1983), 141-155.

[11] Y. Bresler, Bounds on the aliasing error in multidimensional shannon sampling, IEEE Trans. Inf. Theory 42 (1996), 2238-2241.

[12] J. L. Brown, Jr., On the error in reconstructing a nonband-limited function by means of the band pass sampling theorem, J. Math. Anal. Appl. 18 (1967), 75-84. 
[13] G. S. Fang, Whittaker-Kotelnikov-Shannon sampling theorem and aliasing error, J. Approx. Theory 85 (1996), 115-131.

[14] G. H. Hardy and W. W. Rogosinski, Fourier Series, 3rd ed. University Press, Cambridge, 1956.

[15] J. R. Higgins, Sampling Theory in Fourier and Signal Analysis Foundations, Oxford University Press, Oxford, 1996.

[16] H. A. Li and G. S. Fang, On uniform truncation error bounds and aliasing error for multidimensional sampling expansion, Sampl. Theory Signal Image Process. 2 (2003), 103-115.

[17] H. A. Li and G. S. Fang, Sampling theorem of Hermite type and aliasing error on the Sobolev class of functions, Front. Math. China 2 (2006), 252271.

[18] H. G. Feichtinger , K. Gröchenig, Error analysis in regular and irregular sampling theory, Appl. Anal. 50 (1992), 167-189.

[19] Y. Li and G. Feng, Reconstruction of non-bandlimited functions by multidimensional sampling theorem of Hermite type, J. Math. Res. Expo. 29 (2009), 349-354.

[20] M. J. Marsden, F. B. Richards, and S. D. Riemenschneider, Cardinal spline interpolation operators on $\ell^{p}$ date, Indiana Univ. Math. J. 24 (1975), 677689.

[21] S. N. Nikol'skii, Approximation of Functions of Several Variables and Imbedding Theorems, Springer-Verlag, New Yourk, 1975.

[22] Q. I. Rahman, P. Vértesi, On the $L^{p}$ convergence of Lagrange interpolating entire functions of exponential type, J. Approx. Theory 69 (1992), 302-317.

[23] R.L. Stens, A unified approach to sampling theorems for derivatives and Hilbert transforms, Signal Processing 5 (1983), 139-151.

[24] A. F. Timan, Theory of Approximation of Functions of a Real Variable, Pergamon, New York, 1963.

[25] P. Weiss, An estimate of the error arising from misapplication of the sampling theorem , Amer. Math. Sot. Notices 10 (1963), 601-654.

[26] P. Ye and Z. Song, Truncation and aliasing errors for Whittaker-KotelnikovShannon sampling expansion, Appl. Math. J. Chinese Univ. 27 (2012), 412418. 
[27] A. Zygmund, Trigonometric Series, vols. I, II. Cambridge University Press, London, 1968. 